\title{
Neuroticism alters the transcriptome of the frontal cortex to contribute to the cognitive decline and onset of Alzheimer's disease
}

\author{
Céline H. De Jager ${ }^{1}$, Charles C. White ${ }^{1,2}$, David A. Bennett ${ }^{3}$ and Yiyi Ma (1)
}

\begin{abstract}
Accumulating evidence has suggested that the molecular transcriptional mechanism contributes to Alzheimer's disease (AD) and its endophenotypes of cognitive decline and neuropathological traits, $\beta$-amyloid $(A \beta)$ and phosphorylated tangles (TAU). However, it is unknown what is the impact of the AD risk factors, personality characteristics assessed by the NEO Five-Factor Inventory, on the human brain's transcriptome. Using postmortem human brain samples from 466 subjects, we found that neuroticism has a significant overall impact on the brain transcriptome (omnibus $P=0.005$ ) but not the other four personality characteristics. Focused on those cognitive decline related gene co-expressed modules, neuroticism has nominally significant associations $(P<0.05)$ with four neuronal modules, which are more related to PHFtau than $A \beta$ across all eight brain regions. Furthermore, the effect of neuroticism on cognitive decline and AD might be mediated through the expression of module 7 and TAU pathology $(P=0.008)$. To conclude, neuroticism has a broad impact on the transcriptome of human brains, and its effect on cognitive decline and AD may be mediated through gene transcription programs related to TAU pathology.
\end{abstract}

\section{Introduction}

Alzheimer's disease (AD) is the most common form of aging-related dementia, affecting over 5.8 million people in the United States alone (www.alz.org). Many risk factors contributing to AD risk have been identified; some are genetic while others are environmental exposures or life experiences. For some risk factors, e.g., $A P O E \varepsilon 4$, the series of molecular events that lead to the accumulation of $\beta$-amyloid $(A \beta)$ and phosphorylated tangles (TAU), subsequent loss of cognitive function, and, eventually, a diagnosis of dementia has been well documented ${ }^{1}$. However, other risk factors remain poorly characterized at the molecular level. Here, we explored the molecular mechanism linking neuroticism to $\mathrm{AD}$ risk $^{2}$.

\footnotetext{
Correspondence: Yiyi Ma (ym2666@cumc.columbia.edu)

${ }^{1}$ Center for Translational \& Computational Neuroimmunology, Department of Neurology, Columbia University Irving Medical Center, New York, NY 10032, USA

${ }^{2}$ Cell Circuits Program, Broad Institute, 415 Main street, Cambridge, MA 02142, USA

Full list of author information is available at the end of the article
}

Understanding this mechanism may yield important clues to the changes in RNA expression that contribute to the onset of $\mathrm{AD}$ and new avenues for developing therapeutics. Neuroticism, or proneness to distress, is associated with faster cognitive decline and greater $\mathrm{AD}$ risk $^{3}$. By contrast, conscientiousness-being organized, completing purposeful action, and having a drive to achieve-is associated with slower cognitive decline and lower $\mathrm{AD}$ risk $^{3}$. While both have previously been reported to contribute to $\mathrm{AD}^{4,5}$, the role of neuroticism has been more consistently replicated ${ }^{3}$. The molecular composition of these personality traits and the series of molecular events that lead from personality traits to $\mathrm{AD}$ in the brain is unknown.

Recently, large-scale molecular data-such as RNA sequence (RNAseq) data from the dorsolateral prefrontal cortex (DLPFC) ${ }^{6}$-have been generated from the brains of individuals who were deeply characterized while they were alive. Specifically, we repurposed data $^{6}$ generated from 542 participants in two cohort studies of cognitive aging, the Religious Orders Study (ROS) and the

\section{(c) The Author(s) 2021}

(c) (i) Open Access This article is licensed under a Creative Commons Attribution 4.0 International License, which permits use, sharing, adaptation, distribution and reproduction in any medium or format, as long as you give appropriate credit to the original author(s) and the source, provide a link to the Creative Commons license, and indicate if changes were made. The images or other third party material in this article are included in the article's Creative Commons license, unless indicated otherwise in a credit line to the material. If material is not included in the article's Creative Commons license and your intended use is not permitted by statutory regulation or exceeds the permitted use, you will need to obtain permission directly from the copyright holder. To view a copy of this license, visit http://creativecommons.org/licenses/by/4.0/. 
Rush Memory and Aging Project (MAP), which include prospective brain autopsy of each participant ${ }^{7}$. We previously examined the molecular features of the aging cortex that relate to AD pathology and cognitive decline ${ }^{6}$. Here, we investigate the molecular consequences of personality traits leveraging data from the NEO Five Factor personality inventory. The DLPFC is one brain region that has been implicated in neuroticism based on magnetic resonance imaging ${ }^{8}$.

\section{Materials and methods Participants}

The ROS and the Rush Memory and Aging Project (MAP) are longitudinal studies which have enrolled $>3600$ subjects without known dementia at baseline ${ }^{7}$. Both studies were approved by an Institutional Review Board of Rush University Medical Center. All participants signed an informed consent, an Anatomical Gift Act, and a repository consent allowing their data to be repurposed. The participants undergo detailed cognitive testing on an annual basis and other ante-mortem phenotyping including the five personal traits: neuroticism, conscientiousness, extraversion, openness, and agreeableness. These personal variables are measured using 12 items from the NEO Five-Factor Inventory, and each item has scores which range from 0 to 4 and are summed to yield a maximum composite score of 48 , where a higher score indicates greater magnitude of these personal traits ${ }^{5,9-11}$. The two studies are run by the same group of investigators and are designed to be analyzed jointly as the same procedures are used to capture these traits in both studies.

After death, a structured neuropathologic examination is performed to obtain quantitative measures of $A \beta$, TAU, and other pathologies as well as a pathologic diagnosis of $\mathrm{AD}$. $\mathrm{A} \beta$ protein is identified by molecularly-specific immunohistochemistry and quantified by image analy$\mathrm{sis}^{12,13}$. TAU pathology is measured by immunohistochemistry using the AT8 antibody which recognizes a phosphorylated Tau peptide. The values are the percentage area occupied by $A \beta$ using image analysis. TAU proteins represent the density using stereology. Both $A \beta$ and TAU are measured in eight human brain regions: hippocampus, entorhinal cortex, midfrontal cortex, inferior temporal, angular gyrus, calcarine cortex, anterior cingulate cortex, and superior frontal cortex ${ }^{12,13}$, and the mean across the eight brain regions are used as the overall level of $A \beta$ or TAU. Results are transformed by taking the square root to fit a normal distribution. The informed consent was obtained from all subjects and the current analyses was approved by the Institutional Review Board of Columbia University Irving Medical Center.

One hemisphere is frozen at the time of autopsy, and this material was accessed to generate RNAseq data from the DLPFC.

\section{Gene expression modules}

For the current study, there are 466 subjects with both measurements of the brain transcriptome and at least one of the five personality traits. The detailed methods by which the RNAseq were generated and analyzed were described in a prior report ${ }^{6}$. In brief, gray matter from the DLPFC were processed, and RNA was extracted from each sample (Qiagen's miRNeasy mini kit and the RNase free DNAse Set). A cDNA library was prepared from each sample and then sequenced using the Illumina HiSeq platform with $101 \mathrm{bp}$ paired-end reads and an average coverage of 50 million reads. The obtained sequences were aligned to the reference genome of GRCh37 using Bowtie $^{14}$, and the level of expression of individual genes were estimated by the RSEM v1.2.31 ${ }^{15}$ package. This was followed by quantile normalization and batch effect removal using the Combat algorithm ${ }^{16}$. At the quality control stage, those genes with less than 4 reads in 100 individuals are removed, leading to 13,484 genes with expression measures. Groups of co-expressed genes ("modules") across the 542 subjects were defined using the SpeakEasy ${ }^{17}$ consensus clustering algorithm; the 13,484 genes were collapsed into 47 modules of coexpressed genes with an average of 330 genes per module.

\section{Genotype data}

A meta-analysis of the genome-wide association study (GWAS) has reported that 70 single nucleotide polymorphisms (SNPs) from 47 genes were significantly associated with neuroticism ${ }^{18}$. There were 69 SNPs were available in ROSMAP genotype dataset. ROSMAP genotype array and imputations were described in details previously ${ }^{19}$. Briefly, we used the genotype data which were imputed against the Haplotype Reference Consortium reference v1.1. There were 398 subjects with data of genotype, neuroticism trait, and gene expression modules.

\section{Statistical analysis}

As an explorative study design, we used the maximum sample size which was available for each analysis. We applied a generalized linear regression model using the $\mathrm{R}$ "glm" function to conduct the association tests in the study. These models adjust for the covariates of age at death, education, sex, race, study, and technical variables of gene expression experiments such as the experimental batches and RNA integrity score (RIN score). For the mediation analysis, we used the "mediation" $\mathrm{R}$ package (https://cran.rproject.org/web/packages/mediation/ vignettes/mediation.pdf). The outcome variables followed normal distributions and the variances between groups were assumed to be similar. We compared the mediation $P$ value to the direct effect $P$ value. A $P$ value of $<0.05$ is considered as statistical significance. 


\section{Pathway analysis}

Based on the four neuroticism-associated co-expression gene modules $(\mathrm{m} 7, \mathrm{~m} 6, \mathrm{~m} 127$, and $\mathrm{m} 131)$, we identified 20 genes associated with neuroticism (FDR $P<0.05)$. We conducted the pathway enrichment analysis with these 20 using "STRINGdb" (version 10) against the functional Kyoto Encyclopedia of Genes and Genomes (KEGG) Pathway database (https://www.genome.jp/kegg/pathway. html $)^{20}$ with FDR correction ${ }^{21}$. In order to provide an overall view of the general function of the whole module, we also conducted pathway analysis on all the genes included in each of the four neuroticism-associated gene modules.

\section{Results}

\section{Population characteristics}

We first reproduced the role of neuroticism and conscientiousness in the subset of 466 deceased ROSMAP participants (mean of age at death $=88$ years and $62 \%$ female) which have (1) personality inventory data; (2) at least two measures of cognitive performance so that a person-specific slope of cognitive decline can be calculated and (3) transcriptomic data (Table 1). We found that neuroticism contributes to accelerated cognitive decline (beta $=-2.11 \times 10^{-3}, P=3.48 \times 10^{-3}$ )

Table 1 Demographic characteristics of the ROSMAP participants used in the analysis.

\begin{tabular}{lllll}
\hline & Male & Female & All & $P$ \\
\hline Number $^{\mathrm{a}}$ & 179 & 287 & 466 & \\
Age at death (years) $^{\mathrm{b}}$ & $86(6.6)$ & $90(6.6)$ & $88(6.8)$ & $2.56 \mathrm{E}-07$ \\
Education (years) $^{\mathrm{b}}$ & $17.5(3.7)$ & $16.1(3.2)$ & $16.7(3.5)$ & $7.76 \mathrm{E}-05$ \\
White $\left(N\right.$, \%) $^{\mathrm{c}}$ & $176,98 \%$ & $285,99 \%$ & $461(99 \%)$ & 0.59 \\
Neuroticism $^{\mathrm{b}}$ & $16(6.2)$ & $17(6.7)$ & $17(6.5)$ & 0.04 \\
Conscientiousness $^{\mathrm{b}}$ & $33(5.5)$ & $34(4.6)$ & $34(5.0)$ & 0.02 \\
Openness $^{\mathrm{b}}$ & $25(6.1)$ & $26(4.8)$ & $25(5.3)$ & 0.23 \\
Agreeableness $^{\mathrm{b}}$ & $33(4.4)$ & $34(3.5)$ & $34(3.9)$ & 0.08 \\
Extraversion $^{\mathrm{b}}$ & $30(5.9)$ & $30(6.0)$ & $30(6.0)$ & 0.27 \\
Aß pathology $_{\text {(square root) }}$ & $1.3(1.1)$ & $1.6(1.1)$ & $1.5(1.1)$ & 0.05 \\
TAU pathology $_{\text {(square root) }}$ & $1.8(1.3)$ & $2.2(1.2)$ & $2.0(1.3)$ & 0.009 \\
\hline
\end{tabular}

A $\beta$ B-amyloid, TAU abnormally phosphorylated Tau protein, AT8.

${ }^{a} N$ represent the total number of subjects with measurements of any of the five personality traits: neuroticism, conscientiousness, openness, agreeableness, and extraversion.

${ }^{\mathrm{b}}$ Represent the mean and standard deviation of each trait in all subjects, males and females and the $P$ values of their differences between males and females using $t$ test.

${ }^{c}$ Represent the count and percentage of each trait in all subjects, males and females and the $P$ values of their differences between males and females using chi-square test. while conscientiousness is protective against cognitive decline (beta $=2.38 \times 10^{-3}, P=2.58 \times 10^{-2}$ ) controlling for the age at death, sex, race, education and study center. The association with neuroticism remained significant after further controlling for neuropathologic indices such as $A \beta$, Lewy body, and vascular risk factors. However, after controlling for TAU, the association of neuroticism and cognitive decline became borderline (beta $=-1.24 \times$ $10^{-3}, P=5.3 \times 10^{-2}$ ), and it became non-significant when the model is adjusted for all of the neuropathologic indices together (beta $=-9.71 \times 10^{-4}, P=0.11$ ) (Supplementary Table S1). These results are very consistent with prior analyses ${ }^{4,5}$ of other subsets of ROSMAP participants. Having established the role of these two personality traits in aging-related cognitive decline in our sample, we moved on to identify the molecular features which are associated with them.

\section{Neuroticism has an overall impact on modules of co- expressed genes}

We first assessed the impact of neuroticism on the aging DLPFC's transcriptome by conducting an omnibus test, which evaluates the distribution of the associations with all of the 47 groups of co-expressed cortical gene (which we refer to as "modules"). We find that neuroticism has a broad effect on cortical RNA profiles: there is an excess of suggestive associations that are unlikely to have occurred by chance $(P=0.005)$ (Fig. 1A). However, none of the other four personality traits: conscientiousness, agreeableness, openness, extraversion, has an overall impact on the transcriptome $(P>0.05)$ (Supplementary Fig. S1). Another way to illustrate this finding is to illustrate that, at a threshold of $P<0.05$, neuroticism is associated with 18 (38\%) of the 47 cortical modules (Fig. 1B). For comparison, the other four personality traits, together, are only associated with four modules at this threshold (Supplementary Fig. S1). Conscientiousness influences one of these four modules, but this module is unrelated to cognitive decline. Thus, neuroticism has an outsized association with the transcriptome of the aging brain compared to other personality traits.

\section{Neuroticism has an impact on modules of co-expressed genes that are associated with cognitive decline}

We previously reported that 11 modules of coexpressed genes have significant associations with cognitive decline in these data ${ }^{6}$, and four of these modules reached nominal significance for association with neuroticism: modules $\mathrm{m} 7(P=0.02), \mathrm{m} 6(P=0.02), \mathrm{m} 127(P=$ $0.04)$, and $\mathrm{m} 131 \quad(P=0.04)$ (Fig. $1 \mathrm{~B})$. The association between neuroticism and the expression levels of each of the four modules are positive for $\mathrm{m} 7$ and $\mathrm{m} 6$ but negative for $\mathrm{m} 127$ and $\mathrm{m} 131$ (Fig. 1C, D and Supplementary Fig. S2). This means that subjects with more severe 
A

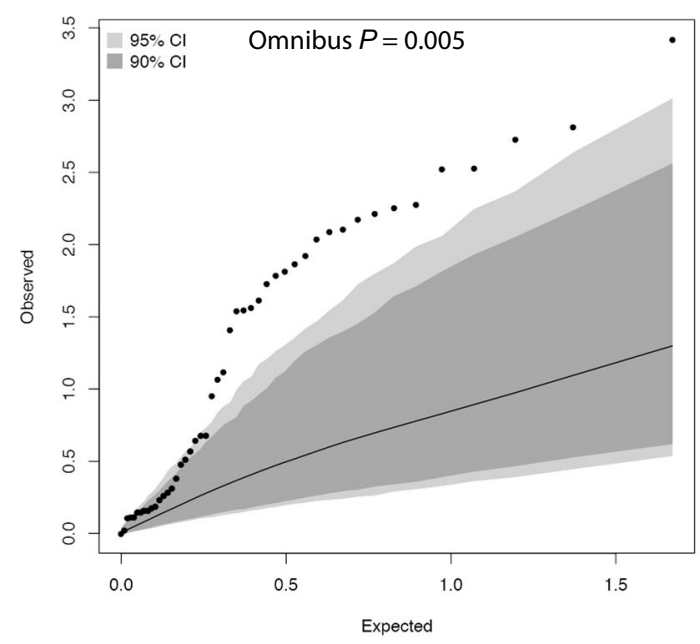

C

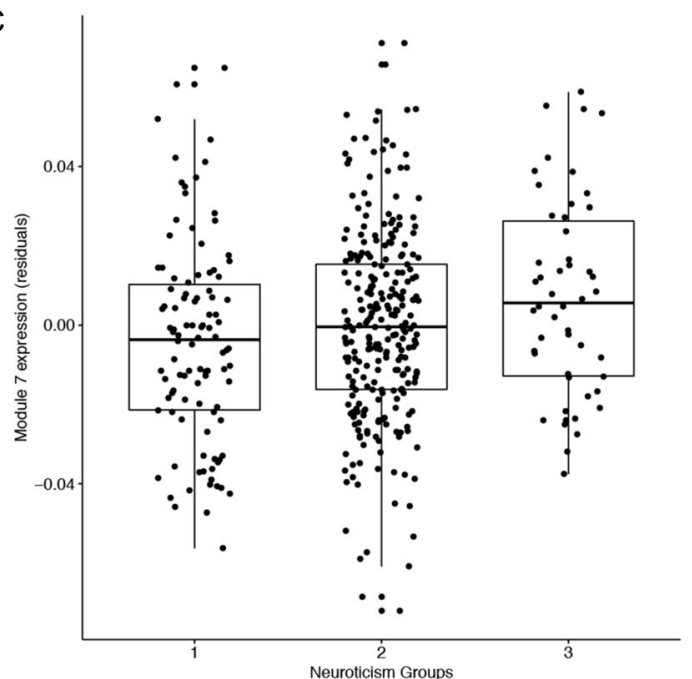

E

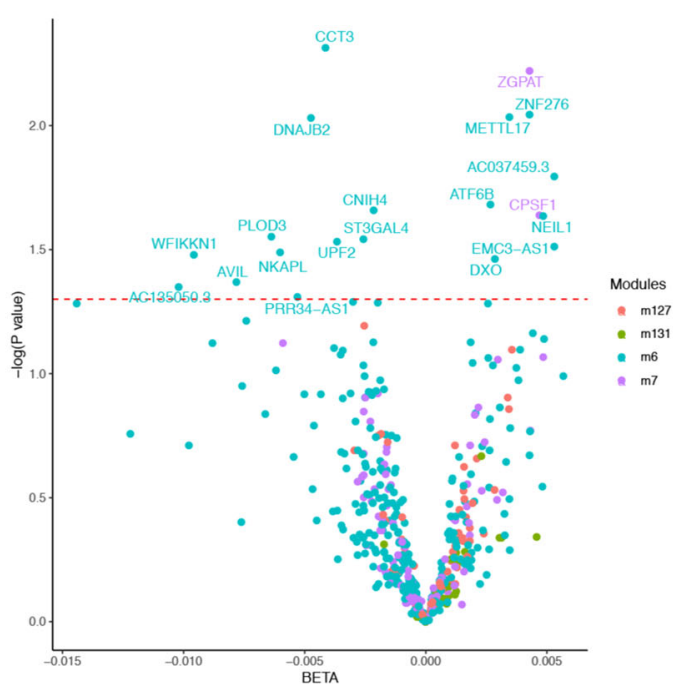

B

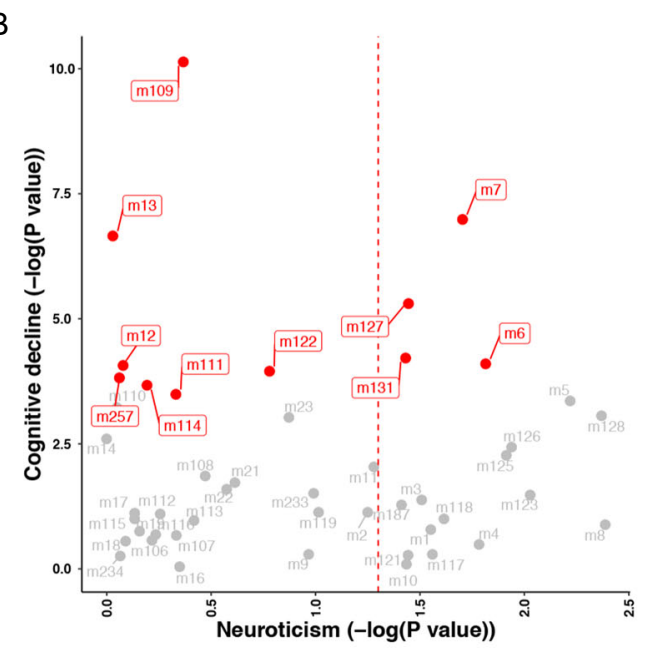

D

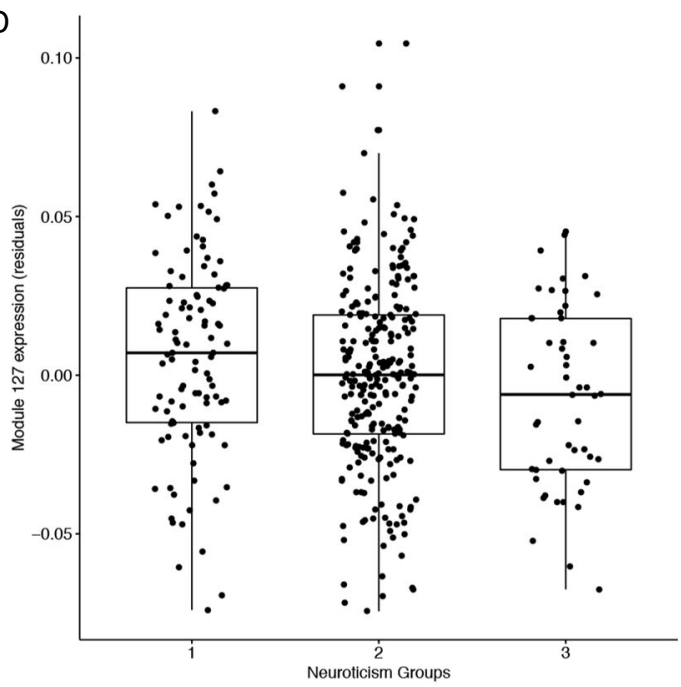

F

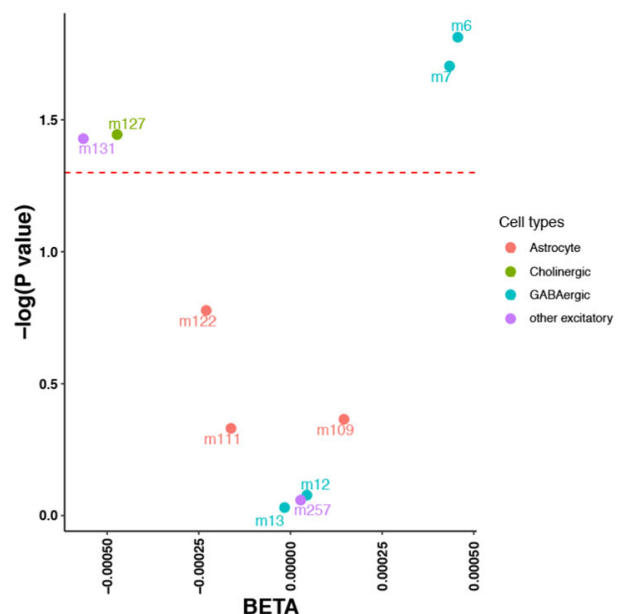

Fig. 1 (See legend on next page.) 
(see figure on previous page)

Fig. 1 Neuroticism with overall or cognitive decline specific cortical co-expression gene modules. A The impact of neuroticism on the overall cortical co-expression gene modules. Each dot represents one module and its expected and observed associations with neuroticism (-log10

$P$ values) are shown on $X$ and $Y$ axis, respectively. The expected $P$ values assume a null distribution with no linear associations between neuroticism score and expression level of co-expressed gene module after adjusting age at death, sex, education, race, postmortem interval, study (ROS or MAP), RNA-seq batch and RIN score. The gray and dark areas indicate the extreme ranges of the QQ plot as generated by chance at a threshold of $P=0.05$ and $P=0.10$, respectively. The 95 and $90 \%$ confidence intervals were empirically derived by randomly assigning participants with the neuroticism score and repeating the analysis 1000 times. Based on the distribution of the observed $P$ values for all the 47 co-expressed gene modules, the overall association between neuroticism and module expression was unlikely to have occurred by chance (omnibus $P=0.005$ ). B The impact of neuroticism on the cognitive decline related co-expression gene modules. Each dot represents one module (the module number is listed next to each dot) and their previously reported $P$ values for the cognitive decline ${ }^{6}$ are shown on the $Y$ axis and the $P$ values $(-\log 10$ transformed) of the effect of neuroticism are shown on the $X$ axis with the red dashed line for the threshold of $P=0.05$. The red dots are those that were previously reported to be associated with cognitive decline ${ }^{6}$. C, D Distribution of cortical module $\mathrm{m} 7$ and $\mathrm{m} 127$ expression in individuals with different levels of neuroticism. Each dot represents one subject, and the subjects are distributed into three groups based on their neuroticism score: Group 1 with score from 0 to 12; Group 2 with score from 13 to 24; Group 3 with score from 25 to 36. On this scale, scores go from 0 (no neuroticism) to 36 (extensive neuroticism). E Volcano plot of the associations between neuroticism score and mRNA expression level of each of the 499 genes included in the top 4 modules associated with both neuroticism and cognitive decline with color codings of $\mathrm{m} 7$ in purple, $\mathrm{m} 6$ in blue, $\mathrm{m} 127$ in green, and $\mathrm{m} 131$ in red. $\mathbf{F}$ Volcano plot of the associations between neuroticism score and the 11 previously reported cognitive decline associated co-expressed gene modules with color codings of different cell type: astrocyte in red, cholinergic neuron in green, GABAergic neuron in blue, and other excitatory neuron in purple. For both volcano plots, $X$ axis shows the BETA and $Y$ axis shows the $-\log 10$ transformed $P$ values with the red dashed line represents the threshold of $P=0.05$.

neuroticism have higher expression levels of the genes included in the $\mathrm{m} 7$ and $\mathrm{m} 6$ modules but lower expression levels of the genes included in $\mathrm{m} 127$ and $\mathrm{m} 131$. We previously reported that subjects with accelerated cognitive decline have higher expression levels of genes in $\mathrm{m} 7$ and $\mathrm{m} 6$ but lower levels in $\mathrm{m} 127$ and $\mathrm{m} 131^{6}$. In this case, the direction of the associations among neuroticism, modules and cognitive decline is consistent, showing that subjects with more severe neuroticism have more rapid cognitive decline, higher expression levels of $\mathrm{m} 7$ and $\mathrm{m} 6$ but lower levels of $\mathrm{m} 127$ and $\mathrm{m} 131$. Thus, we prioritized a set of modules that may be responding to greater neurotic behavior and cause downstream cognitive decline. However, none of the 11 modules display a relationship to conscientiousness, which is protective for $\mathrm{AD}$; that process may work through other mechanisms or may work through another brain region.

We further conducted the association tests with the individual gene included in the modules $\mathrm{m} 7, \mathrm{~m} 6, \mathrm{~m} 127$, and m131 to identify those individual genes which are related to neuroticism. Out of the 499 genes in these 4 modules, 20 have FDR $P<0.05,18$ of which are in $\mathrm{m} 6$ and the remaining 2 are in $\mathrm{m} 7$ (Fig. 1E). The pathway enrichment analysis for these 20 genes highlights the involvement of mRNA surveillance pathway (FDR_ $P=0.008$, hits $=2$ $(2 \%))$ and protein processing in endoplasmic reticulum $($ FDR_P $=0.01$, hits $=2(1 \%))$ (Table 2). In order to have a general understanding of the neuroticism-associated modules, we also added the pathway analysis (Supplementary Table S2) for all the genes included in each module and the top pathway for $\mathrm{m} 7$ was MAPK signaling pathway $\left(\right.$ FDR_P $P=6.27 \times 10^{-4}$, hits $\left.=5(2 \%)\right)$ and the top pathway for m6, m127 and 131 was the same, which was the metabolic pathways (FDR_P $=0.03,9.36 \times 10^{-4}$, and
$7.92 \times 10^{-4}$, hits $=13(1 \%), 8(0.7 \%)$ and $5(0.4 \%)$ for $\mathrm{m} 6$, $\mathrm{m} 127$ and 131, respectively).

We also annotated the cell types which may express the four modules associated with both neuroticism and cognitive decline (Fig. 1F). All of the four modules are annotated to be expressed in neurons. Both modules $\mathrm{m} 7$ and $\mathrm{m} 6$ are enriched in the genes expressed by GABAergic neurons, while $\mathrm{m} 127$ is enriched in genes expressed by cholinergic neurons and $\mathrm{m} 131$ is enriched in genes expressed by other excitatory neurons.

\section{Neuroticism and cognitive decline associated co-expressed gene modules are related to $A D$ pathology}

We further analyzed which AD pathology, $A \beta$ or TAU, are primarily involved in neuroticism pathology. Neuroticism has a positive association with TAU $(P=0.03)$ but not $\mathrm{A} \beta(P=0.16)$ in this subsample of participants (Fig. 2A), both of these measures are calculated as the mean densities across eight different brain regions. We then repeated our analysis by replacing the neuroticism trait with the 69 SNPs which were reported to be associated with neuroticism in GWAS and were available in ROSMAP participants ${ }^{18}$. Only one SNP was associated with $\mathrm{A} \beta$, which was rs2073498 in the RASSF1 locus (BETA $=0.238, \mathrm{SE}=0.106, P=0.026$ ). There were three SNPs with significant associations with TAU, which were rs7199949 at PRSS53 (BETA $=-0.172, \mathrm{SE}=0.086, P=$ $0.046)$, rs240780 at $A S C C 3(\mathrm{BETA}=0.223, \mathrm{SE}=0.083$, $P=0.007)$, and rs3173615 at TMEM106B (BETA $=0.22$, $\mathrm{SE}=0.083, P=0.008$ ). Thus, overall, we have observed that neuroticism was associated with TAU pathology more than with $A \beta$ pathology.

For the four modules associated with both neuroticism and cognitive decline, three of them have smaller $P$ values 
Table 2 Pathway enrichment analysis.

\begin{tabular}{llllll}
\hline Term_id & Proteins & Hits & $\boldsymbol{P}$ value & FDR & Term_description \\
\hline 3015 & 86 & 2 & 0.0004 & 0.008 & mRNA surveillance pathway \\
4141 & 162 & 2 & 0.001 & 0.013 & Protein processing in endoplasmic reticulum \\
601 & 26 & 1 & 0.009 & 0.047 & Glycosphingolipid biosynthesis_lacto and neolacto series \\
514 & 31 & 1 & 0.011 & 0.047 & Other types of O-glycan biosynthesis \\
3410 & 33 & 1 & 0.011 & 0.047 & Base excision repair \\
5030 & 49 & 1 & 0.017 & 0.051 & Cocaine addiction \\
310 & 50 & 1 & 0.017 & 0.051 & Lysine degradation \\
\hline
\end{tabular}

for TAU than the $P$ values for $A \beta$ based on our previously reported associations ${ }^{6}$ and only module $\mathrm{m} 7$ was reported to have significant association with the average level of TAU (Fig. 2B). More detailed analysis with the eight region-specific measures of TAU and $A \beta$ also suggest that the effect of module $\mathrm{m} 7$ on TAU outperformed all of the other tested associations (Fig. 2C).

\section{Relationship between neuroticism and module 7 gene expression}

As noted above, the neuroticism GWAS $^{18}$ reported that there were 70 SNPs associated with neuroticism, and these 70 SNPs are annotated as involving 47 discrete genes in susceptibility to neuroticism. Although none of the 47 genes were found in $\mathrm{m} 7$ (data were not shown), rs739134 in the C22orf46 locus was significantly associated with both neuroticism $(\mathrm{BETA}=1.545, \mathrm{SE}=0.56, P=0.006)$ and module 7 gene expression $(\mathrm{BETA}=0.005, \mathrm{SE}=0.002, \quad P=0.016)$ (Fig. 3A), indicating a possible causal effect of neuroticism on m7. However, rs739134 cannot be treated as the instrumental variable to infer the causal effect of neuroticism on $\mathrm{m} 7$ because the effect of rs739134 on $\mathrm{m} 7$ remains significant after adjusting for neuroticism (BETA $=0.0045, \mathrm{SE}=0.002$, $P=0.037$ ), indicating that this variant may have pleiotropic effects; it may affect both $\mathrm{m} 7$ and neuroticism through different pathways (Fig. 3B). Thus, we cannot conduct the mendelian randomization to infer the suggested causal effect of neuroticism on $\mathrm{m} 7$.

\section{Mediation modeling to propose a sequence of events}

Using a rigorous statistical methodology and our crosssectional data obtained from autopsy material, we assessed the most likely location of each module in the sequence of events leading to $\mathrm{AD}$ to help to generate hypotheses that can be tested in preclinical and in vitro models. Mediation modeling suggests that the four modules of co-expressed genes which are altered in expression in the frontal cortex by neuroticism may contribute to accelerating aging-related cognitive decline. For example, using $\mathrm{m} 7$ which is the module most strongly associated with cognitive decline, the model in which neuroticism is associated with faster cognitive decline $(P=0.008)$ through an association with higher $\mathrm{m} 7$ expression is more significant than the alternative model where neuroticism has a direct causal effect on cognitive decline which bypasses $\mathrm{m} 7$ expression $(P=0.042)$ (Fig. 3 left). We find similar results for modules $\mathrm{m} 6$ and $\mathrm{m} 127$ but not m131 (Supplementary Table S3).

Based on our previous and current report that $\mathrm{m} 7$ is the module most directly contributing to the accumulation of TAU pathology ${ }^{6}$, we added this variable to our model to more precisely evaluate the mechanism (Fig. 3 right). Thus, we propose that the most likely scenario in terms of the role of $\mathrm{m} 7$ in neuroticism's effect on AD is the following sequence of events: more neuroticism $\rightarrow$ more $\mathrm{m} 7$ in the frontal cortex $\rightarrow$ more TAU pathology accumulation $\rightarrow$ more cognitive decline $\rightarrow$ greater likelihood of developing AD dementia. $\mathrm{m} 127$ and $\mathrm{m} 131$ are not associated with TAU pathology and may therefore work through another mechanism to mediate the effects of neuroticism on cognitive decline (mediation $p<0.01$ ).

\section{Discussion}

Neuroticism appears to have a broad impact on the biology of the older brain, as measured by RNA expression: $\sim 1 / 5$ of our modules of co-expressed genes are associated with an individual's level of neuroticism at a nominal $p<0.05$ threshold. Thus, we have started to map out the molecular changes in the target organ (brain) that relate to neuroticism, an important personality trait that is a risk factor for $\mathrm{AD}$ and aging-related cognitive decline. The molecular substrate of neuroticism remains poorly understood as few studies have systematically assessed this question in large numbers of individuals, so this report is an important step forward in demonstrating a widespread biological effect of neuroticism in the frontal cortex. These large changes in older individuals beg the question of whether the changes are clinically meaningful: are they associated with symptoms, with changes in cognition? 

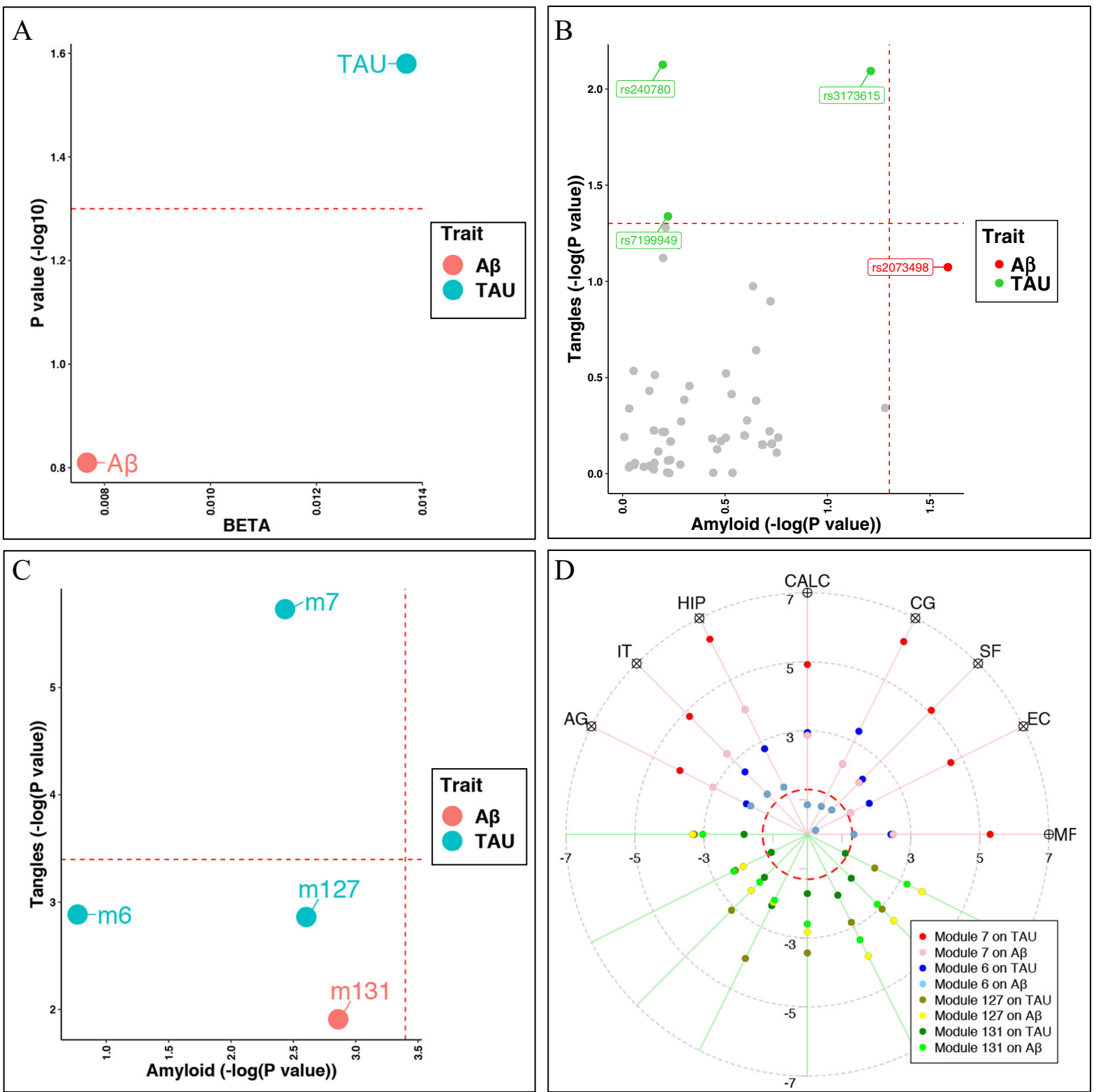

Fig. 2 Neuroticism and cognitive decline associated co-expressed gene modules are more related to TAU than $\mathbf{A} \boldsymbol{\beta}$. A Effect of neuroticism on the average levels of brain TAU and $A \beta$, which were defined as the mean of the measurements across eight different brain regions of midfrontal cortex (MF), entorhinal cortex (EC), superior frontal cortex (SF), anterior cingulate cortex (CG), calcarine cortex (CALC), hippocampus (HIP), inferior temporal cortex (IT), and angular gyrus (AG). $X$ axis represent the BETAs and $Y$ axis represent the $-\log 10$ transformed $P$ values for the neuroticism score on either TAU (blue dot) or A (red dot) after adjusting for the age at death, sex, postmortem interval, race, education, study (ROS or MAP). The horizontal red dashed line represents the threshold of $P=0.05$. B Comparisons of the associations to A $\beta$ vs. TAU for the neuroticism-associated SNPs reported to by Nagel 2918. Each dot represented one SNP with the $P$ values ( $-\log 10$ transformed) for A $(X$ axis) and TAU ( $Y$ axis). Gray dots showed those SNPs with $P>0.05$ for both $A \beta$ and TAU. Red dots showed the SNP significantly associated with $A \beta(P<0.05)$ but not TAU $(P>0.05)$, while green dots those SNPs significantly associated with TAU $(P<0.05)$ but not A $(P>0.05)$. C Comparisons of the associations to A $\beta$ vs. TAU for the four modules related to both cognitive decline and neuroticism. Each dot represents one module with the $P$ values $(-\log 10$ transformed) reported by Mostafavi et al. ${ }^{6}$ for $A \beta$ ( $X$ axis) and TAU (Y axis). Red dots show those modules with smaller reported $P$ value for $A \beta$ than TAU while blue dots show those modules with smaller reported $P$ value for TAU than A . D Target plot shows the associations between the four cognitive decline/neuroticism related modules and measures of A $\beta$ and TAU across eight different brain regions, which are displayed counter-clockwise from MF, EC, SF, CG, CALC, HIP, IT, and AG. Each dot on each axis represent the signed $P$ value (- $\log 10$ transformed) of the association between each module and each measures of either $A \beta$ or TAU at each brain region with the color codings of: module $m 7$ on TAU in red and $A \beta$ in pink, m6 on TAU in blue and $A \beta$ in skyblue, m127 on TAU in gold and $A \beta$ in yellow, and m131 on TAU in darker green and A $\beta$ in green. The red dashed circle represents the threshold of $P=0.05$. The covariates include age at death, sex, postmortem interval, race, education, study (ROS or MAP), RIN and RNA experimental batch. A $\beta$ $\beta$-amyloid, TAU abnormally phosphorylated Tau protein, AT8. 

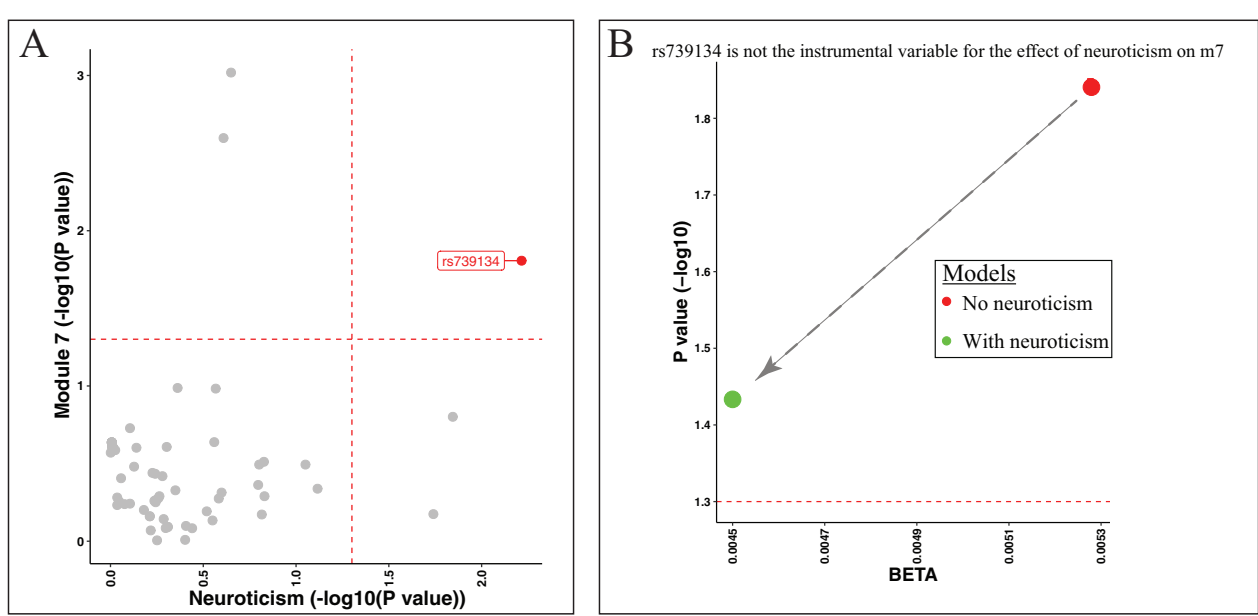

C

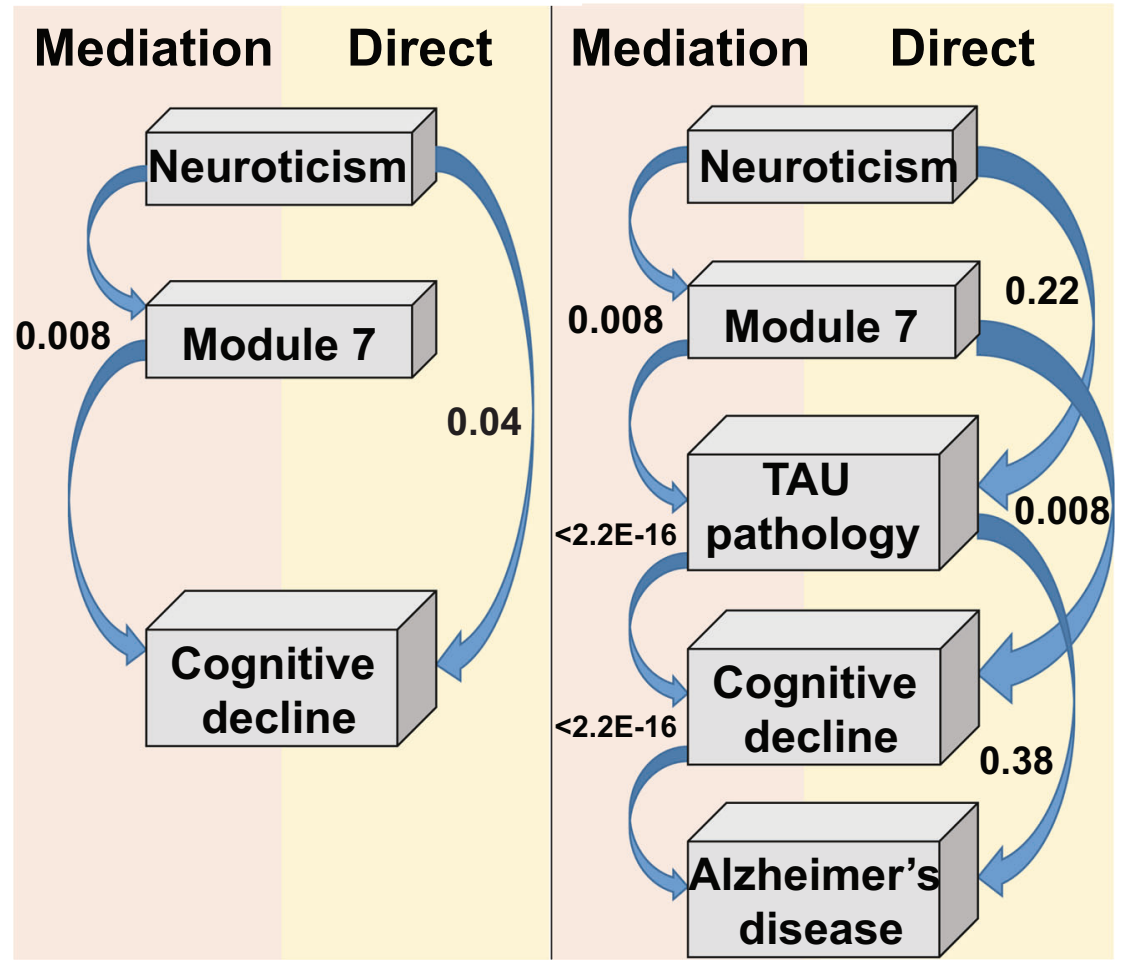

Fig. 3 Causal chain analysis by mendelian randomization and mediation tests. A Rs 739134 was associated with both neuroticism and module 7 expression. Each dot represented one of the 69 reported neuroticism-associated SNPs (Nagel 2918) and their $P$ values (-log 10 transformed) for neuroticism ( $X$ axis) and module 7 expression ( $Y$ axis) in ROSMAP were presented. B Rs739134 is not the instrumental variable for the effet of neuroticism on module 7 gene expression. The effects of rs 739134 on the module 7 expression for the were presented by BETA ( $X$ axis) and $P$ values ( $-\log 10$ transformed) in the two models without the adjustment of neuroticism (red dot) and with the adjustment of neuroticism (green dot). The red dashed horizontal line represented the significance threshold of $P=0.05$. C Module $\mathrm{m} 7$ mediates the effect of neuroticism on cognitive decline. Left panel: we present the most likely sequence of events based on our cross-sectional data. The most likely scenario is that being more neurotic leads to an increase in $\mathrm{m} 7$ which then contributes to an acceleration in cognitive decline. The $p$ value for this 0.008 while, in the same analysis, the direct effect of neuroticism on cognitive decline is much less $(P=0.04)$. Not shown is an analysis which shows that $\mathrm{m} 7$ is much more likely to be downstream of neuroticism than upstream where an increase in $\mathrm{m} 7$ would make someone more neurotic. Right panel: we expand the model shown in the left panel with Tau pathology which is known to immediately precede cognitive decline and a diagnosis of AD which occurs after someone has begun to decline. Here, three mediation tests are presented: (1) Neuroticism $\rightarrow \mathrm{m} 7 \rightarrow$ Tau is more likely than a direct effect of Neuroticism $\rightarrow$ Tau, (2) $\mathrm{m} 7 \rightarrow$ Tau $\rightarrow$ Cognitive decline is more likely than $\mathrm{m} 7 \rightarrow$ Cognitive decline, and (3) Tau contributes to AD dementia by affecting the slope of cognitive decline (Tau $\rightarrow$ Cognitive decline $\rightarrow$ AD), which is the well-accepted model of AD. TAU abnormally phosphorylated Tau protein, AT8. 
We explored this question in detail and found that three of the modules associated with neuroticism are also associated with the slope of cognitive decline and a diagnosis of $\mathrm{AD}$, providing a potential molecular link between $\mathrm{AD}$ and its risk factor, neuroticism. Since association studies of cross-sectional data, such as the one that we performed, cannot formally resolve the direction of causality, we used rigorous statistical methodology to test for mediation which enables us to propose a sequence of events that best fits our cross-sectional data. We considered deploying mendelian randomization methods to address this question which can leverage the 70 SNPs reported to be associated with neuroticism; however, the lack of SNP associations with module expression prevented this. The one SNP in the C22orf46 locus that shows some level of effect on both traits appears to have a pleiotropic effect; thus a genetic approach to this question was not informative. Nonetheless, our mediation analyses are very useful in generating hypotheses that can then be tested in cell culture or mouse model systems. In our analyses, we find that module $\mathrm{m} 7$, the module with the strongest association with neuroticism is most likely to increase in expression in response to increased neurotic behavior and to then contribute to cognitive decline (Fig. 3). In addition, we have found that the neuroticism was related to TAU pathology more than $A \beta$, which was in line with the previous GWAS report ${ }^{18}$ that one of the neuroticism susceptibility loci includes $M A P T$, the gene encoding tangles. These insights are important because, if validated in future studies, it could guide the development of future drugs to prevent AD. For example, a drug reducing $\mathrm{m} 7$ expression may be ineffective in preventing neuroticism, but it may be helpful to prevent the spread of negative molecular changes in the cortex that lead to cognitive decline and AD. Since personality traits like neuroticism cannot be readily changed, blocking the dysfunctional consequences of such personality traits may be the best option with which to manage individuals at risk of AD because of their personality traits.

We focused on neuroticism given that it is the personality trait most strongly associated with risk of AD and that its association has been well validated. Nonetheless, we also evaluated four other major personality traits in our analyses to be thorough, and, while we found some associations, they were much fewer than the neuroticism associations and did not relate to cognitive decline. It is possible that the effects of these other personality traits may be more subtle and/or that we need to sample other brain regions to identify their effects.

Our study has many strengths, including its large sample size and largely community-based design, which facilitates repurposing our results and may offer insights translatable to the older community. We also have very deep RNAseq profiles, which enhanced the modules' information content. Nonetheless, there are important limitations as well: we have already discussed the crosssectional nature of the data that is a practical limitation of autopsy studies and prevents us from formally resolving the issue of causality. In addition, the average age at death was 88 years, which may make it challenging to extrapolate our results to the population of younger older individuals where many treatments for $\mathrm{AD}$ will need to be targeted.

Overall, we have prioritized a set of molecular changes in the frontal cortex that are involved in both neuroticism and aging-related cognitive decline and $\mathrm{AD}$. We have also proposed a specific sequence of events that can now be tested in model systems. This hypothesis can be refined by performing many of the analyses reported here at the gene level instead of at the level of the module of co-expressed genes: this will facilitate validation studies and will be necessary to enable drug development efforts by presenting a specific target. We have therefore laid a strong foundation for future biological investigations of the effects of an important AD risk factor that affects many individuals: neuroticism.

\section{Acknowledgements \\ We are grateful to the participants in the Religious Order Study, the Rush Memory and Aging Project. This work is supported by the US National Institutes of Health U01AG61356, RP30AG10161, R01AG17917, R01AG15819. Y.M. is supported by the 2019-AARF-644521.}

\section{Author details}

${ }^{1}$ Center for Translational \& Computational Neuroimmunology, Department of Neurology, Columbia University Irving Medical Center, New York, NY 10032, USA. ${ }^{2}$ Cell Circuits Program, Broad Institute, 415 Main street, Cambridge, MA 02142, USA. ${ }^{3}$ Rush Alzheimer Disease Center, RUSH University Medical Center Chicago, IL 60612, USA

\section{Author contributions}

C.H.D. and Y.M.: study concept and design, data analysis and manuscript draft. C.C.W.: guidance of omnibus test. D.A.B.: provide data and critical review of manuscript.

\section{Data availability}

All the data and analysis output are available via the AD Knowledge Portal (https://adknowledgeportal.synapse.org). The AD Knowledge Portal is a platform for accessing data, analyses, and tools generated by the Accelerating Medicines Partnership (AMP-AD) Target Discovery Program and other National Institute on Aging (NIA)-supported programs to enable open-science practices and accelerate translational learning. The data, analyses and tools are shared early in the research cycle without a publication embargo on secondary use. Data is available for general research use according to the following requirements for data access and data attribution (https://adknowledgeportal. synapse.org/DataAccess/Instructions). The link to the data for this manuscript is https://adknowledgeportal.synapse.org/Explore/Studies/DetailsPage? Study=syn3219045.

\section{Conflict of interest}

The authors declare no competing interests.

\section{Publisher's note}

Springer Nature remains neutral with regard to jurisdictional claims in published maps and institutional affiliations. 
Supplementary information The online version contains supplementary material available at https://doi.org/10.1038/s41398-021-01253-6.

Received: 8 April 2020 Revised: 6 January 2021 Accepted: 28 January 2021 Published online: 24 February 2021

\section{References}

1. Theendakara, V., Peters-Libeu, C. A., Bredesen, D. E. \& Rao, R. V. Transcriptional effects of ApoE4: relevance to Alzheimer's disease. Mol. Neurobiol. 55, 5243-5254 (2018).

2. Wilson, R. S., Begeny, C. T., Boyle, P. A., Schneider, J. A. \& Bennett, D. A. Vulnerability to stress, anxiety, and development of dementia in old age. Am. J. Geriatr. Psychiatry 19, 327-334 (2011).

3. D'lorio, A. et al. Meta-analysis of personality traits in Alzheimer's disease: a comparison with healthy subjects. J. Alzheimers Dis. 62, 773-787 (2018).

4. Wilson, R. S. et al. Premorbid proneness to distress and episodic memory impairment in Alzheimer's disease. J. Neurol. Neurosurg. Psychiatry 75, 191-195 (2004)

5. Wilson, R. S., Schneider, J. A., Arnold, S. E., Bienias, J. L. \& Bennett, D. A. Conscientiousness and the incidence of Alzheimer disease and mild cognitive impairment. Arch. Gen. Psychiatry 64, 1204-1212 (2007).

6. Mostafavi, S. et al. A molecular network of the aging human brain provides insights into the pathology and cognitive decline of Alzheimer's disease. Nat. Neurosci. 21, 811-819 (2018).

7. Bennett, D. A. et al. Religious orders study and rush memory and aging project. J. Alzheimers Dis. 64, S161-S189 (2018).

8. Lucas, I., Balada, F., Blanco, E. \& Aluja, A. Prefrontal cortex activity triggered by affective faces exposure and its relationship with neuroticism. Neuropsychologia 132, 107146 (2019).
9. Krueger, K. R., Wilson, R. S., Shah, R. C., Tang, Y. \& Bennett, D. A. Personality and incident disability in older persons. Age Ageing 35, 428-433 (2006).

10. Wilson, R. S. et al. Neuroticism, extraversion, and mortality in a defined population of older persons. Psychosom. Med 67, 841-845 (2005).

11. Buchman, A. S. et al. Neuroticism, extraversion, and motor function in community-dwelling older persons. Am. J. Geriatr. Psychiatry 21, 145-154 (2013).

12. Bennett, D. A., Schneider, J. A., Arvanitakis, Z. \& Wilson, R. S. Overview and findings from the religious orders study. Curr. Alzheimer Res. 9, 628-645 (2012).

13. Bennett, D. A. et al. Overview and findings from the rush Memory and Aging Project. Curr. Alzheimer Res 9, 646-663 (2012).

14. Langmead, B., Trapnell, C., Pop, M. \& Salzberg, S. L. Ultrafast and memoryefficient alignment of short DNA sequences to the human genome. Genome Biol. 10, R25 (2009).

15. Li, B. \& Dewey, C. N. RSEM: accurate transcript quantification from RNA-Seq data with or without a reference genome. BMC Bioinforma. 12, 323 (2011).

16. Johnson, W. E., Li, C. \& Rabinovic, A. Adjusting batch effects in microarray expression data using empirical Bayes methods. Biostatistics 8, 118-127 (2007).

17. Gaiteri, C. et al. Identifying robust communities and multi-community nodes by combining top-down and bottom-up approaches to clustering. Sci. Rep. $\mathbf{5}$, 16361 (2015).

18. Nagel, M. et al. Meta-analysis of genome-wide association studies for neuroticism in 449,484 individuals identifies novel genetic loci and pathways. Nat Genet 50, 920-927 (2018).

19. Felsky, D. et al. Neuropathological correlates and genetic architecture of microglial activation in elderly human brain. Nat. Commun. 10, 409 (2019).

20. Szklarczyk, D. et al. STRING v10: protein-protein interaction networks, integrated over the tree of life. Nucleic Acids Res $\mathbf{4 3}$, D447-D452 (2015).

21. Benjamini, Y. H. Y. Controlling the false discovery rate: a practical and powerful approach to multiple testing. J. R. Stat. Soc. 57, 289-300 (1995), 